\title{
Die Einsamkeit des Arztes und der «lebendige Drang nach Geschichte». Zum historischen Selbstverständnis der Medizin bei Richard Koch
}

Urban Wiesing

\section{Summary}

This article tries to identify the theory of medical history in the work of Richard Koch (1882-1949). Rejecting the wide-spread notion of medicine as a science he vigorously argued for a self-image of medicine as an art. From that point of view he built up his theory of medical history and historiography. $\mathrm{He}$ wanted medical historiography to be part of practical medicine.

\section{Zusammenfassung}

Es wird der Versuch unternommen, die Theorie der Medizingeschichte im Werk von Richard Koch (1882-1949) darzustellen. Im Gegensatz zur weitverbreiteten Ansicht der Medizin als einer Wissenschaft argumentierte Richard Koch für ein Selbstverständnis der Medizin als einer Kunst. Von diesem Standpunkt her entwickelte er seine Theorie der Geschichte und Historiographie der Medizin. Er betrachtete die medizinische Historiographie als Teil der praktischen Medizin.

\section{Einleitung}

Die Medizin in Deutschland vollzog im frühen zwanzigsten Jahrhundert zumindest auf theoretischer Ebene eine merkliche Wandlung. Hochrangige Fachvertreter der akademischen Medizin distanzierten sich öffentlich von der Überzeugung, die Medizin fände ihr Heil, sofern sie sich nur als Natur-

Priv.-Doz. Dr. med. Dr. phil. Urban Wiesing, Institut für Theorie und Geschichte der Medizin, Universität Münster, Waldeyer Str. 27, D-48149 Münster 
wissenschaft oder angewandte Naturwissenschaft umgestalte. Jenes euphorisch verfolgte Programm, das die Medizin seit der Mitte des 19. Jahrhunderts nachhaltig geprägt hatte, sah sich von mehreren Seiten mit Kritik konfrontiert. Im Einklang mit anderen, gleichfalls als bedrohlich eingeschätzten Entwicklungen des gesellschaftlichen und kulturellen Lebens verbreitete sich das Schlagwort von der «Krise der Medizin», vornehmlich in Deutschland. Im Rahmen dieser Neuorientierung stellte sich gleichermassen die Frage nach dem geschichtlichen Selbstbild der Medizin. Das fortschrittsoptimistische, positivistische Geschichtsverständnis der Naturwissenschaften, das die Medizin für sich übernommen hatte, stand in Zweifel, als die Medizin ihre epistemologische Eigenständigkeit betonte. Überdies bemerkten die Ärzte, dass sie sich zu Unrecht gänzlich von ihrer eigenen Vergangenheit glaubten verabschieden zu können. Ein neues, ein nicht-positivistisches Geschichtsbild musste für die Medizin gefunden werden.

Richard Koch (1882-1949) ${ }^{1}$ gehörte zu den Medizintheoretikern der ersten Hälfte des 20. Jahrhunderts, die den Glauben an eine Medizin als Naturwissenschaft erschüttert haben. Ausführlich begründet vertrat er die These, die Medizin sei von Haus aus eine Kunst im antiken Verständnis einer téchne. Unter dieser Prämisse musste er sich unvermeidlich auch zum geschichtlichen Selbstverständnis der Medizin äussern. Es sei hier seine Antwort dargestellt, wie ein vertretbares historisches Selbstbild der Medizin aussehen und was «Geschichte» für Ärzte bedeuten könnte.

\section{Problemstellung: Medizin und Geschichte im frühen 20. Jahrhundert}

Mit dem enthusiastischen Aufbruch der Medizin in der zweiten Hälfte des 19. Jahrhunderts, mit dem Glauben, sie sei angewandte Naturwissenschaft und nur in diese Richtung umzugestalten, hatte die Medizin auch das positivistische Geschichtsverständnis der Naturwissenschaften übernommen. Dieser Vorgang war einerseits zwar mit einem Gewinn an Historizität verbunden, da das Objekt der Naturwissenschaften, die Natur, zuletzt in Orientierung am Darwinismus historisiert wurde. Andererseits verschwand jeder historische Aspekt aus dem Wissen über die Natur. Wissenschaft und Wis-

1 Zu Richard Koch siehe Preiser, G. (Hg.) (1988) Richard Koch und die ärztliche Diagnose. Hildesheim; Rothschuh, K. E. (1980) Richard Hermann Koch (1882-1949) 1. Teil, Zur Biographie. Medizinhistorisches Journal 15: 16-43, 2. Teil, Werk und Würdigung, S. 223-243; Schwann,H. (1982) Richard Kochs Beziehungen zum Karl-Sudhoff-Institut. Archivstudie anläßlich seines 100. Geburtstages. NTM-Schriftenreihe 19:94-103; Wiesing, U. (1197) Richard Koch: Auschwitz - Eine Feier für die Seelen der Toten. In: C. Wiesemann, A. Frewer (Hg.) Medizin und Ethik im Zeichen von Auschwitz. 50 Jahre Nürnberger Ärzteprozeß. Erlangen, Jena, S. 99-103 und S. I-XVI; Wiesing, U. (1997) Medizin und Moral bei Richard Koch. Zeitschrift für Ethik in der Medizin 9: 134-150. 
senschaftsgeschichte trennten sich gänzlich. «[...] Progreßbegeisterung und Gegenwartsbewußtsein traten in den Vordergrund; [...] Von nun an sollte die Geschichtsschreibung der Naturwissenschaften im Grunde keine Sache rezenter Forscher mehr sein.» Die Naturwissenschaften nahmen durchaus in Kauf, «daß die Verbindung zur allgemeinen Geschichtswissenschaft wie Geschichtsphilosophie» ${ }^{3}$ abgebrochen wurde - und die Medizin tat es ihnen gleich: Auch ihre Vergangenheit verkam zur einer mehr oder weniger belanglosen Vorgeschichte ohne Bezug zur Gegenwart.

Doch der Glaube, als Naturwissenschaft das «endgültige» epistemologische Selbstverständnis der Medizin gefunden zu haben, erwies sich sehr bald als trügerisch. Spätestens in den zwanziger Jahren bezweifelten zahlreiche Ärzte das Wissenschaftsverständnis des 19. Jahrhunderts ${ }^{4}$. Für dieses Umdenken boten sich plausible Argumente an: Der naturwissenschaftlich orientierten Medizin waren zunächst nur wenige praktische Erfolge beschieden. Neue Therapien waren eher dem Zufall als systematischer wissenschaftlicher Forschung geschuldet. Gegen zahlreiche, weit verbreitete Krankheiten fehlten wirksame Mittel. Eine rationelle und überdies effektive Therapie ergab sich keinesfalls von selbst, wie es die Protagonisten einer «naturwissenschaftlichen Medizin» stets verkündet hatten. Es mehrten sich ferner die Klagen über zu häufige und voreilige Anwendungen neuer Therapien, auch von renommierten Klinikern. Die psychischen Aspekte des Krankseins und der Arzt-Patient-Beziehung drohten in Vergessenheit zu geraten. Überhaupt wurde die ärztliche Praxis mit minderer Aufmerksamkeit bedacht, ganz im Gegensatz zur Tätigkeit im Labor. Victor v. Weizsäcker (1886-1957) sprach in diesem Zusammenhang von einem «törichten Hyperlaboratismus» ${ }^{5}$. Und die Patienten liefen in Scharen zu anderen Heilkundigen ${ }^{6}$.

2 Engelhardt, D.v. (1977) Naturwissenschaft und Geschichtlichkeit in der Neuzeit. Fortschritte der Medizin 95: 2203-2205, S. 2205.

3 Engelhardt, D.v. (1983) Verzeitlichung der Natur und Enthistorisierung der Naturerkenntnis in der Biologie der Neuzeit. In: Biohistorisch Instituut der Rijksuniversiteit Utrecht (Hg.) De complementariteit van geschiedenis en wijsbegeerte der biologie. Utrecht, S. 5-22, S. 15; siehe auch ders. (1979) Historisches Bewußtsein in der Naturwissenschaft von der Aufklärung bis zum Positivismus. Freiburg, München; ders. (1987) Wissenschaftsgeschichte auf den Versammlungen der Gesellschaft Deutscher Naturforscher und Ärzte 1822-1972. Stuttgart.

4 Neben Koch sind zu dieser Richtung Ernst Schweninger (1850-1924), Louis R. Grote (1886-1960), Georg Honigmann (1863-1930), Erwin Liek (1878-1935) und andere zu zählen; Henk ten Have spricht von einer «epistemologischen Tradition» in der Philosophie der Medizin, die in den dreissiger Jahren von der «anthropologischen Tradition» abgelöst wurde; ten Have, H. (1995) The Anthropological Tradition in the Philosophy of Medicine. Theoretical Medicine 16:3-14.

5 Weizsäcker, V.v. (1923) Über Gesinnungsvitalismus. Klinische Wochenschrift 2: 30-33, S. 32.

6 Datenmaterial zu nichtärztlichen Heilberufen liefert Wuttke-Groneberg, W. (1982) «Kraft im Schlagen, Kraft im Ertragen!» - Medizinische Reformbewegung und Krise der Schulmedizin in der Weimarer Republik. In: H. Cancic (Hg.) Religions- und Geistesgeschichte der Weimarer Republik. Düsseldorf, S. 277-300. 
Die «Krise der Medizin» 7 im frühen zwanzigsten Jahrhundert und die Neubesinnung sind in mehrfacher Hinsicht nicht ohne die Geschichte der Medizin zu verstehen. So waren es unter anderem Medizinhistoriker, die mit Rückgriff auf vergangene Zeiten die euphorischen Gewissheiten des späten 19. Jahrhunderts in Zweifel zogen. Doch der Blick zurück in die Vergangenheit sollte angesichts zerstörter Dogmen gleichermassen kompensierende Orientierung leisten. Ferdinand Sauerbruch (1875-1951) bestätigte in seiner berühmten Düsseldorfer Rede von 1926 die sowohl kritische als auch potentiell prophylaktische Funktion der Medizingeschichte:

«Geschichtliche Betrachtungsweise hätte [...] uns selbst vor Überhebung, Überschätzung und Irrwegen geschützt. [...] Es ist sicher kein Zufall, daß am deutlichsten und klarsten den Zwiespalt der heutigen Medizin Geschichtsforscher [...] erfaßt haben.» ${ }^{8}$

Und Max Neuburger (1868-1955) stand nicht allein, als er der Geschichte der Medizin in diesem Zusammenhang sogar eine krisenbewältigende, eine «aufbauende» Funktion zuordnete:

«Noch vor wenigen Jahrzehnten schien der Abbruch der Tradition endgültig, der Zusammenhang zwischen alter und neuer Medizin für immer aufgehoben zu sein. Heute dagegen räumen es die führenden Männer willig ein, daß bei der Zertrümmerung des historisch Gewordenen auch viel an kostbaren Werten zugrunde ging, ja der gesamte wissenschaftliche Werdeprozeß drängt geradezu gebieterisch dahin, das, was die Alten vermutet haben, auf der Basis moderner Biologie wieder aufzubauen.»9

Mit dieser Aussage kamen der Medizinhistoriographie nicht nur neue Funktionen zu, sie sah sich gleichermassen mit einem Problem konfrontiert: Unweigerlich musste sie ein neues, plausibleres historisches Selbstverständnis der Medizin benennen. Wenn der «Abbruch der Tradition» nicht mehr «endgültig» sein sollte, wenn der «wissenschaftliche Werdeprozeß» «gebieterisch»

7 Siehe zusammenfassend Klasen, E.M. (1984) Die Diskussion über eine «Krise» der Medizin in Deutschland zwischen 1925 und 1935. Med. Diss., Mainz; Bothe, D. (1991) Neue Deutsche Heilkunde 1933-1945. Dargestellt anhand der Zeitschrift «Hippokrates» und der Entwicklung der volksheilkundlichen Laienbewegung. Husum, insb. S. 16-37; Haug, A. (1985) Die Reichsarbeitsgemeinschaft für eine Neue Deutsche Heilkunde (1935/36). Ein Beitrag zum Verhältnis von Schulmedizin, Naturheilkunde und Nationalsozialismus. Husum; in Anlehnung an Haug Baader, G. (1984) Die Medizin im Nationalsozialismus. Ihre Wurzeln und die erste Periode ihrer Realisierung 1933-1938. In: Ch. Pross, R. Winau (Hg.) Nicht mißhandeln. Das Krankenhaus Moabit 1920-1945. Berlin, S. 61-107, insb. S. 81-88; Kater, M.H. (1989) Die Krise der Ärzte und der Medizin im Dritten Reich. In: Ch. Pross, G. Aly (Hg.) Der Wert des Menschen. Medizin in Deutschland 1918-1945. Berlin, S. 357-371. Die Arbeiten beschäftigen sich auch mit der eigentümlichen Assimilation des Gedankengutes durch die Medizin im Nationalsozialismus; unter diesem Aspekt siehe auch Thomann, K.-D. (1985) Auf dem Weg in den Faschismus. Medizin in Deutschland von der Jahrhundertwende bis 1933. In: B. Bromberger, H. Mausbach, K.-D. Thomann (Hg.) Medizin, Faschismus und Widerstand. Drei Beiträge. Köln, S. 15-185.

8 Sauerbruch, F. (1926) Heilkunst und Naturwissenschaft. Vortrag gehalten auf der 89. Naturforscherversammlung. Die Naturwissenschaften 14: 1081-1090, S. 1090.

9 Neuburger, M. (1926) Die Lehre von der Heilkraft der Natur im Wandel der Zeiten. Stuttgart, S. 1. 
in die Vergangenheit wies, dann musste man eine andere Verlaufsform des «Werdeprozesses» beschreiben. Denn das fortschrittsgläubige, positivistische Geschichtsverständnis hatte gerade nicht in die Vergangenheit, sondern einzig in die Zukunft verwiesen.

Überdies erkannten zumindest einige Ärzte eine ungelegene Eigenschaft des positivistischen Geschichtsverständnisses: Es diskreditiert nicht nur die Vergangenheit, sondern auch die Gegenwart, sobald sie nur vergangen ist. Auf diesen Makel für Ärzte, die nach bleibendem Ruhm trachteten, verwies beispielsweise Bernd Aschner (1883-1960):

«Wenn die heutigen Kliniker die Medizin vor 100 Jahren als unwissenschaftlich und unkritisch ansehen, dann wird man unsere Leistungen in weiteren 100 Jahren als ebenso belanglos betrachten. Damit wären wohl die wenigsten heutigen Universitätslehrer einverstanden.»10

So gesehen stand das positivistische Geschichtsverständnis aus vielerlei Gründen in Zweifel. Offensichtlich ging es in der Medizin nicht nur kontinuierlich und steil bergauf, offensichtlich hatte man Altes, Bewahrenswertes vergessen, offensichtlich führte der Glaube, die Gegenwart sei aller Vergangenheit überlegen, zu Unstimmigkeiten - ein neues Geschichtsbild musste beschrieben werden.

Die Zweifel am positivistischen Geschichtsbild der Medizin unterstützten einen Verweis auf die überhistorischen Konstanten in der Medizin, also auf das gegenteilige Extrem. Nicht mehr das stets wachsende und sich vermehrende naturwissenschaftliche Wissen sollte das geschichtliche Selbstverständnis der Medizin prägen, sondern die überzeitlichen, die unveränderten, da unveränderlichen Merkmale der Medizin. In diesem Zusammenhang ist von einer verklärenden Beschwörung der «ewigen» Werte, der «wahren» ärztlichen Persönlichkeit und von einem Neo-Hippokratismus zu berichten: Die seit Urzeiten unveränderliche Persönlichkeit des «wahren» Arztes, vorbildhaft von Hippokrates gelebt, präge das Wesen der Medizin ${ }^{11}$. Die geschichtsphilosophischen Einwände gegen solche Vorstellungen sind leicht zu ersehen: Denn im Grunde verabschiedete man die Geschichte aus der Geschichtsphilosophie, da man nicht das geschichtlich Gewordene als wesensbestimmend für die Medizin erachtete, sondern das Übergeschichtliche,

10 Aschner,B. (1933) Die Krise der Medizin. Lehrbuch der Konstitutionstherapie. 5. Aufl., Stuttgart, Leipzig, S. 44. Bereits Schweninger hat in seinem für die ganze Richtung initialen Buch auf diese Eigenschaft des positivistischen Geschichtsverständnisses verwiesen: «Was weißt denn Du [gemeint ist der Wissenschaftler], was die Gelehrten von morgen oder übermorgen über Deine heutige Auffassung sagen werden?» (Schweninger, E. (1906) Der Arzt. Frankfurt a. M., S. 126).

11 Siehe Wiesing, U. (1996) Die Persönlichkeit des Arztes und das historische Selbstverständnis der Medizin. Zur Medizintheorie von Ernst Schweninger, Georg Honigmann und Erwin Liek. Medizinhistorisches Journal 31:181-209 
Ewige, Zeitinvariante. Gleichwohl, der Verweis auf den «wahren» und damit zugleich «ewigen» Arzt als geschichtsprägende Konstante der Medizin war ausgesprochen populär.

Die Diskussion um das historische Selbstverständnis der Medizin bewegte sich zwischen beiden Extremen: Auf der einen Seite das positivistische Geschichtsbild, das von einem steten Fortschritt ausging und die Vergangenheit als weithin bedeutungslose Vorstufe abqualifizierte, auf der anderen Seite der Verweis auf Ewiges, Unveränderliches, das die Medizin seit jeher kennzeichnete und zu dem es zurückzufinden galt. Es sei nun untersucht, wie Richard Koch auf die geschichtsphilosophische Herausforderung seiner Zeit reagierte.

\section{Richard Kochs Medizintheorie}

Das Geschichtsbild der Medizin ist bei Richard Koch nur im Rahmen seines medizintheoretischen Gesamtwerkes zu verstehen. Deshalb seien vorab wichtige Thesen dargestellt. Neben den Ausführungen zum Vitalismus konzentrierte sich Koch vor allem auf die Wissenschaftstheorie der Medizin. Stets widersprach er dem seit der zweiten Hälfte des 19. Jahrhunderts dominierenden epistemologischen Selbstverständnis der Medizin: Die Medizin könne sinnvollerweise gar nicht den Status einer Naturwissenschaft oder einer angewandten Naturwissenschaft beanspruchen, sondern sei eine Kunst, eine téchne.Auch wenn die Medizin naturwissenschaftliche Erkenntnisse und Methoden nutze, so bleibe ihr Status doch eigenständig, denn die Zielsetzungen seien unterschiedlich. Die Naturwissenschaften seien primär der selbstzweckhaften Erkenntnissuche verpflichtet, aus der allenfalls vermittelt Nutzen erwachsen könne. Der Medizin gehe es in erster Linie nicht um Erkenntnis, sondern um effektive Handlung im Einzelfall. Als Disziplin konstituiere sich die Medizin nur durch die Übernahme einer Aufgabe.

Koch nutzt die ureigene Verpflichtung der Medizin - die Handlung im Krankheitsfalle - als Ausgangspunkt, um die epistemologische Frage der Medizin zu beantworten. Er will eine Theorie der Medizin entwerfen, die «den Gegenstand da zu fassen [...] [sucht], wo er sich in seiner Natürlichkeit zeigt und wo deutlich wird, daß es sich in der Medizin um eine elementare Betätigung des Menschen handelt $»^{12}$. Koch sucht nicht bei den Naturwissenschaften, sondern im traditionellen normativen Gefüge der ärztlichen Tätigkeit nach Ursprung und Einheit der Medizin.

12 Koch, R. (1926) Der Anteil der Geisteswissenschaften an den Grundlagen der Medizin. Archiv für Geschichte der Medizin 18: 273-301, S. 300. 
«[...] Medizin ist uns heute auf einer ursprünglichen Beziehung gegründet zwischen der nur teilweise in ihren Einzelheiten erkannten und erkennbaren Ganzheit eines kranken Menschen und der Ganzheit eines helfenden Menschen, der seine Mittel nicht nur der Naturwissenschaft entnimmt, sondern allem, das solche Mittel zu liefern imstande ist.» ${ }^{13}$

Da er den Begriff «Wissenschaft» einschränkend den «Wissensordnungen vorbehält, deren Ziel die Erkenntnis ist» ${ }^{14}$, urteilt er über den wissenschaftstheoretischen Status der Medizin:

«Hieraus ergibt sich, daß die Heilkunde nicht Naturwissenschaft, überhaupt nicht Wissenschaft sein kann. Ihr Zweck ist nicht Erkenntnis, sondern Befriedigung eines Bedürfnisses. Sie will kranken Menschen nützen.» ${ }^{15}$

Was könnte man unter einer Medizin als Wissenschaft allenfalls noch verstehen? Kochs Antwort: «Wenn man die Medizin selbst Wissenschaft nennt, meint man, daß sie sich eines geordneten Wissens bedient. ${ }^{16}$ Gleichwohl, die Medizin habe eine Aufgabe übernommen, sei eine menschliche Tätigkeit, «eine besonders geschulte Art der Hilfe» ${ }^{17}$. Infolgedessen habe eine Medizintheorie die zentralen Begriffe und Strukturen ärztlichen Handelns zu erläutern, um den Auftrag der Medizin möglichst effektiv umzusetzen. Dies verpflichte die Medizin auf einen eklektischen Pragmatismus: Es ist unerheblich, aus welchem Umfeld theoretischer Gedanken eine Therapie kommt; wenn sie hilft, dann soll der Arzt sie nutzen: «Gut ist, was nützt, auch wenn man nicht weiß, wieso es nützt.» ${ }^{18}$

Indem Richard Koch die Medizin als eine téchne beschreibt, hat er wissentlich auf ein epistemologisches Selbstverständnis zurückgegriffen, das keineswegs neu, sondern seit der Antike bekannt war.

«Wir bemerken, daß wir auch heute das Wesen der Heilkunde nicht anders sehen können, als wie es uns schon in den hippokratischen Schriften entgegentritt. Alles, was hinzukommt, ist akzidentell.» ${ }^{19}$

Damit ist die geschichtsphilosophische Herausforderung nicht mehr zu übersehen. Wenn das Wesen der Medizin bereits in der Antike zutreffend

13 Koch,R.(1928) Die Geschichte der Medizin im Universitätsunterricht.Archiv für Geschichte der Medizin 20: 2-16, S. 5.

14 Koch 1926 (Anm. 12), S. 290.

15 Koch, R. (1920) Die ärztliche Diagnose. Beitrag zur Kenntnis des ärztlichen Denkens. 2. überarb. Aufl., Wiesbaden, S. 58-59 (1. Aufl. 1917).

16 Koch, R. (1921) Die Bedeutung der Geschichte der Medizin für den Arzt. Fortschritte der Medizin 35: 217-225, S. 220

17 Koch, R. (1930) Der Begriff der Medizin. In: Philosophische Grenzfragen der Medizin (= Vorträge für Geschichte der Medizin an der Universität Leipzig, Bd. 3). Leipzig, S. 9-31, S. 30 .

18 Koch, R. (1921) Laien-Medizin. Frankfurter Zeitung und Handelsblatt Nr. 508, S. 1-2, S. 1.

19 Koch 1920 (Anm.15), S. 64. «Wo auch die Ursprünge unserer abendländischen Medizin liegen mögen, in großem Schriftwerk niedergelegt ist diese auf Beobachtung und Vernunft gegründete von aller Dämonenangst freie Heillehre seit der hippokratischen Zeit» (Koch, R. (1927) Medizin-Historik. Frankfurter Zeitung und Handelsblatt Nr. 685, S. 1-2, S. 2). 
bestimmt wurde und nicht erst in der zweiten Hälfte des 19. Jahrhunderts, dann ist das dominierende Geschichtsbild der Medizin unzutreffend - mit weitreichenden Konsequenzen: Das historische Selbstverständnis muss neu beschrieben werden, die gesamte Fortschrittsmetaphorik hat sich neu zu orientieren, und die Vergangenheit, zuvor als grundsätzlich unbedeutend abqualifiziert, erlangt einen enormen Zuwachs an Bedeutsamkeit. Dieser Wandel im Geschichtsverständnis präge - so Koch - seine Zeit:

«[...]; daß sich aber heute die bedeutendsten Aerzte um der lebendigen Medizin willen und nicht aus antiquarischem Interesse mit der Geschichte ihres Faches beschäftigen, ist eine Erscheinung so seltsamer Art, von so tiefgreifender, man darf ruhig aussprechen, von so epochemachender Bedeutung, daß man nicht daran vorbeisehen darf, wenn man sich nicht künstlich um das Verständnis der Zeit bringen will.» ${ }^{20}$

Diese Erscheinung wäre wohl kaum «epochemachend», begrenzte sie sich einzig auf die Medizin. Die Zweifel am positivistischen Geschichtsbild würden - so Koch - überdies aus ganz anderen kulturellen Bereichen genährt. Angesichts einer Ausstellung über die «Kunst der Irren» in Frankfurt a.M. 1921 äussert er sich zum Wandel im Geschichtsverständnis, den die Kunst bewirkt habe:

«Alle Verblüffung über fremde, primitive Kunst stammt ebenso wie die Verblüffung über die Kunst der Irren aus einer Täuschung, in der befangen wir Kulturmenschen leben. Wir glauben an geradlinigen Fortschritt in der Richtung der Fähigkeiten, die die Westwelt auszeichnet. Eben lehrt uns das Leben, daß es diesen geradlinigen Fortschritt nicht gibt, nicht geben soll.» ${ }^{21}$

Bemerkenswerterweise bezieht sich Koch in den beiden letzten Zitaten auf das «Lebendige»: «Um der lebendigen Medizin willen» habe sich das Augenmerk auf die Geschichte gelegt, und «das Leben» habe nun gerade eines der wirkmächtigsten Vorurteile der westlichen Welt entlarvt. In einer Bemerkung zum Fortschritt verweist Koch gleichermassen auf das «Lebendige» und zerstört mit dieser Eigenschaft das übliche Bild von «Fortschritt». «Entwicklung ist nicht gradlinig. Fortschritt ist ein Wort, das anf [!] das, was es bezeichnen soll, nicht recht paßt. Es geht nicht immer in einer Linie geradeaus im Lebendigen. ${ }^{22}$ Bei diesen Anleihen aus dem Bereich des Biologischen stellt sich zwangsläufig die Frage, inwieweit der zeitliche Ablauf von Ereignissen mit derartigen Metaphern zutreffend beschrieben ist und in welcher Weise Vergangenheit demgemäss dargestellt werden kann.

20 Koch 1927 (Anm. 19), S. 1.

21 Koch, R. (1921) Die Kunst der Irren. Frankfurter Zeitung und Handelsblatt Nr. 57, S. 1.

22 Koch, R. (1921) Die Bedeutung der Geschichte der Medizin für den Arzt. Fortschritte der Medizin 35: 217-225, S. 219. 


\section{«Lebendige Geschichte» und historische Erkenntnismöglichkeiten}

Und in der Tat, Koch nutzt die biologische Metaphorik nicht nur für die Medizin, sondern gleichermassen für den zeitlichen Ablauf von Ereignissen in der Medizin. Die Medizinhistoriographie habe sich «des organischen Werdens der gesamten Heilkunst ${ }^{23}$ zu widmen. Die Eigenschaften der zeitlichen Abfolge von Ereignissen implizieren weitreichende Konsequenzen: Die Geschichte, nicht nur die der Medizin, sei gestaltbar, denn es gelte,

«daß die Geschichte nicht so abläuft, wie sie aus notwendig wirkenden Ursachen ablaufen muß, sondern daß wir sie selbst nach einem frei gewählten Bilde gegen alle Notwendigkeit gestalten können.» ${ }^{24}$

Gleichwohl: Auch wenn der Ablauf von Ereignissen in der Zeit nicht determiniert ist und ein Moment der Freiheit die Geschichte bestimmt, so lassen sich trotzdem Entwicklungen abstrakt beschreiben, wenn auch nicht mit der Präzision naturwissenschaftlicher Gesetze. «Es besteht eine gewisse Wahrscheinlichkeit, daß auch im Geschichtlichen aus gleichen Umständen sich gleiche Zustände entwickeln.» ${ }^{25}$ Die Eigenschaften des zeitlichen Ablaufs der Ereignisse, ihre «Lebendigkeit» und die Freiheit der Gestaltung, bestimmen - so Koch - gleichermassen die Erkennbarkeit des Vergangenen.

«Wir haben gelernt, daß wir weit weniger wissen können wie fremdes und früheres Leben gewesen ist, als wir uns das früher dachten. Vergangenheit bleibt vergangen, auch wenn sie noch so glänzend dargestellt wird. Unvergänglich und zuverlässig bleiben die Dokumente, Gegenstände und Schriften.» ${ }^{26}$

Koch vergleicht die Geschichte in ihrer «Lebendigkeit» mit einer Arzt-Patient-Beziehung. Damit schlägt er eine Brücke zwischen dem Tun eines Arztes und dem eines Historikers. «Wie man sich als Arzt oder sonst als ein Tätiger mit dem Individuellen auseinandersetzt, das wird niemals in starren Regeln überlieferbar sein.» ${ }^{27}$ Wenn der zeitliche Ablauf von Ereignissen aufgrund seiner «Lebendigkeit» nur begrenzt in wissenschaftlichen Regeln zu beschreiben ist, was verbleibt dem Historiker? Er kann sie nur individuell nacherleben. Koch bezieht sich hier auf eine Unterscheidung von Heinrich Rickert (1863-1936), die wiederum auf Wilhelm Windelband (1848-1915) zurückgeht, derzufolge die Geisteswissenschaften «ideographische» Aussagen treffen könnten, während die Naturwissenschaften $\mathrm{zu}$ «nomotheti-

23 Koch 1928 (Anm. 13), S. 4.

24 Koch, R. (1923) Ärztliches Denken. Abhandlungen über die philosophischen Grundlagen der Medizin. München, S. VIII; Koch spricht aus diesem Grunde von Paracelsus, dass er «als geschichtsbildender Mensch» (Koch 1927 [Anm. 19], S. 2) gewirkt hätte.

25 Koch 1921 (Anm. 22), S. 224.

26 Koch 1928 (Anm. 13), S. 16.

27 Koch 1921 (Anm. 22), S. 223. 
schen» Aussagen befähigt seien ${ }^{28}$. Daraus folgt für die Geschichtsschreibung: Sie könne, anders als die Naturwissenschaften, keine Gesetze auffinden, sondern müsse das Wesen erfassen. «Die Methode der Geschichte ist also weder Deduktion noch Induktion, sondern eben das Nacherleben des Gewesenen.» ${ }^{29}$ Überdies ist die Darstellung der Vergangenheit zwangsläufig mit Wertungen verbunden und niemals wirklich objektiv. Sie ist stets für einen bestimmten Adressaten zu schreiben. Koch trifft in seinem gesamten Werk in diesem Punkt eine entschiedene Wahl: «Geschichte ist für Menschen geschrieben, Fachgeschichte für Fachmenschen, Medizingeschichte für Aerzte. $»^{30}$

Was folgt aus den Eigenschaften der Geschichte für das historiographische Arbeiten? Das Wesen vergangener Epochen liesse sich nicht erfassen so Koch -, indem alle Einzelheiten aufgelistet würden. Insofern ergeben sich mit Bezug auf die empirische Basis zwei idealtypische Extreme der historischen Darstellung: die - vermeintlich - exakte Reproduktion der Vergangenheit durch unendliche Detailvielfalt und der Mythos, der nur aus der Rezeption eines vergangenen Ereignisses besteht. Für Koch ist klar, dass beide Darstellungsformen ihrem Zweck in der Medizin nicht gerecht werden und dass «Geschichte als Mythos, wie als Reproduktion der Vergangenheit ihren Höhepunkt überschritten hat $»^{31}$. Gleichwohl - und abermals mit Verweis auf das «Lebendige»:

«Geschichte, die im Leben der Menschen eine Rolle spielen soll, soll kein Mythos sein, aber von unserer gegenwärtigen Geschichte können wir behaupten, daß sie dem Mythos etwas näher rücken muss, wenn sie Anspruch auf Lebendigkeit erhebt.» ${ }^{32}$

\section{Die Wurzeln der Medizin in den biologischen Eigenschaften der Organismen}

Um Kochs Vorstellungen zum zeitlichen Ablauf von Ereignissen in der Medizin vertiefen zu können, sind weitere Details seiner Medizintheorie zu bedenken. Wie gesehen, stellt Koch die Arzt-Patient-Beziehung und deren normative Ausrichtung in den Mittelpunkt aller Erwägungen. Ausgehend vom Phänomen des Helfens errichtet er seine Medizintheorie. Zudem schreibt er der Medizintheorie die Aufgabe zu, die historischen Züge des ärztlichen Tuns zu erkunden:

28 Vergl. Windelband,W. (1894) Geschichte und Naturwissenschaft. Rektoratsrede. Strassburg; Rickert, H. (1913) Die Grenzen der naturwissenschaftlichen Begriffsbildung. Tübingen, 2. Aufl.

29 Koch 1921 (Anm. 22), S. 219.

30 Koch 1921 (Anm. 22), S. 220.

31 Koch 1928 (Anm. 13), S. 16.

32 Koch 1921 (Anm. 22), S. 219. 
«Sie [die Theorie der Medizin] hat sich zunächst mit dem Vorhandensein dieses Helfens zu beschäftigen. Sie hat zu beschreiben, wo es unter den einfachsten Verhältnissen zuerst nachweisbar ist, und unter welchen Einflüssen es sich bis zu seiner Form, die es heute hat, wandelte.»33

Allein die vorrangige Stellung der historiographischen Aufgabe im Gebäude seiner Medizintheorie verweist auf ihre hohe Bedeutung; und nicht zufällig beginnt Koch in seinem medizintheoretischen Hauptwerk, der «ärztlichen Diagnose» von 1917/1920, mit einem historischen Rückblick. Aber warum erhofft er sich von einer Bewältigung der medizinhistorischen Aufgabe einen hohen Gewinn für seine Medizintheorie? Eine solche Forschung ist nur fruchtbar, wenn ihre Ergebnisse zum Selbstverständnis der Medizin etwas beitragen können, d.h. wenn Erkenntnisse vom Ursprung der Medizin zugleich Erkenntnisse vom Wesen der Medizin liefern. Und genau davon geht Koch aus.

Bei seinem Rückgriff auf die Vergangenheit überschreitet Koch den Bereich menschlicher Bemühungen um die Gesundheit und geht zurück «auf primitive Zustände und auf das allerprimitivste [...], auf das spontane krankheitsabwehrende Verhalten des Körpers selbst» ${ }^{34}$. «Deshalb führt die Geschichte der Heilkunde über den Urmenschen zurück zu dem Verhalten des kranken Tieres, zurück zu der Reaktion des geschädigten, lebenden Protoplasmas.» ${ }^{35}$ Hier sieht Koch einen Vorläufer der Medizin. Wie Organismen reagieren, wenn sie schädlichen Einflüssen ausgesetzt sind, entspricht im Prinzip dem Wesen der Medizin; sie übernimmt damit eine Aufgabe mit biologischen Vorstufen: «Die Heilkunde hat ihre Wurzeln in dem nützlichen Reagieren von Organismen auf Schädigungen. $»^{36}$ Insofern baut die Medizin die krankheitsabwehrenden biologischen Reaktionen im Bereich des bewussten menschlichen Handelns weiter aus.

Diese Genealogie birgt allerdings einige Probleme, die der Klärung bedürfen und überdies das Geschichtsverständnis der Medizin berühren. Ärztlich zu handeln ist nur sinnvoll, sofern die Hoffnung begründet ist, die Natur zu übertreffen, also erfolgreicher zu sein als das spontane krankheitsabwehrende Verhalten. Anderenfalls würde sich die Mühe nicht lohnen. Diese Hoffnung - so Koch - sei jedoch berechtigt:

«Wir glauben vor der Tatsache zu stehen, daß unsere Vernunft der Vernunft des Organismus unter manchen Umständen überlegen ist. Aus diesem Verhältnis wird Medizin. » $^{37}$ 
Der Grund für die optimistische Einschätzung liegt in der Eigenschaft der spontanen biologischen Reaktionsweisen. Koch unterstellt den Organismen neben einer Vernunft auch eine gewisse Unvernunft. Die spontanen krankheitsabwehrenden Reaktionen müssten nicht immer nützlich sein, sie könnten sich als unzureichend oder gar als schädlich erweisen. Insofern gilt: Auch wenn die Medizin in den natürlichen Reaktionen der Organismen auf Reize ihren Vorläufer findet, handelt ein Arzt streng genommen gegen die Natur, da auch unzureichende oder schädliche biologische Reaktionen zur «Natur» eines Organismus gehören. Die Medizin führt einerseits die «nützlichen Reaktionen eines Organismus auf Schädigungen» im Bereich des menschlichen Bewusstseins fort, andererseits handelt sie wider die Natur. Hierbei erweist es sich freilich als klug, die Reaktionen der Natur zu nutzen.

«Der größte Teil unserer hochentwickelten Medizin beruht auf der vernünftigen Nutzbarmachung dieser Eigenschaft lebendigen Gewebes.» ${ }^{38}$

Zurück zum Geschichtsverständnis: Koch sieht in der Medizin ein verfeinertes Reiz-Reaktionsschema, eine mit Bewusstsein ausgebaute, ursprüngliche Reaktion lebendiger Wesen. Was lässt sich nun aus dem Wissen um die biologischen Vorläufer der Medizin gewinnen?

«Man kann über das Verhalten bewußter Wesen hinaus zu dem Verhalten der lebendigen, reizbaren Substanz als solche gelangen und von hier aus durch Rückwärtsverfolgung des zurückgelegten Weges bis zum bewußten Wesen, schließlich bis zum Menschen als Arzt eines anderen Menschen, erkennen, daß trotz Überbauung der einfachsten Reizübertragung und -Verwertung mit immer höheren Übertragungsbögen, deren letzter das Denkhirn des Menschen ist, es sich immer um dieselbe Sache gehandelt hat.» ${ }^{39}$

Der historisch-genetischen Rekonstruktion zufolge handelt es sich bei dem Phänomen «Medizin» um etwas in seinem Kern weithin Unverändertes, «immer um dieselbe Sache». Die historische Nachforschung führt demnach zum Wesenhaften der Medizin und verweist auf die Konstanten in der Entwicklung der Medizin - im Prinzip macht der Arzt nichts anderes als ein Organismus bei schädigenden Reizen. Was resultiert daraus für das Geschichtsbild? Hier verfolgt Koch die Hypothese, dass sich im Ursprung der Medizin ihr eigentliches Wesen bereits herauskristallisiert hätte und dass ihre weitere Geschichte davon geprägt sei. Die Herausforderungen der Medizin, der Drang nach Reaktion, nach Handeln, die Situation, dass etwas angesichts einer Veränderung geschehen muss, daran habe sich - so Koch - kaum etwas

38 Koch, R. (1920/21) Die ärztliche Diagnose. Beitrag zur Kenntnis des ärztlichen Denkens. Zweite, umgearbeitete Auflage [Selbstanzeige]. Annalen der Philosophie 2: 496-498, S. 496; an anderer Stelle: «In Wirklichkeit ist aber alles Gesundwerden so überwiegend ein Werk der Natur, daß der primitive und der zivilisierte Mensch nur wenig hat hinzufügen können. Dieses Wenige aber ist kostbarster Menschenbesitz» (Koch 1927 [Anm. 19], S. 1).

39 Koch 1920/21 (Anm. 38), S. 496. 
geändert. Somit begründet er mit der biologischen Genealogie die Beständigkeit bestimmter Strukturen in der Medizin im Verlauf der Zeit.

\section{Die Klassiker der Medizin}

In anderem Zusammenhang erläutert Koch noch einmal das Verhältnis von Wechselndem und Konstantem in der Geschichte der Medizin, und zwar bei der Frage, warum bestimmte Schriften zu Klassikern geworden sind. Was zeichnet sie aus, dass ihnen diese Eigenschaft zukommt? Koch untersucht diese Frage an den Hippokratischen Aphorismen, und er weiss um die Tragweite seines Unterfangens: «Es ist eine Frage, die den Kern der Medizin trifft, warum denn diese Aphorismen zu klassischer Bedeutung gelangt sind.»40 Koch zieht durchaus in Betracht, dass die Aphorismen unterschiedlich interpretiert und gewertet wurden. Auch ihr Inhalt begründe die ungewöhnliche Rezeption nur unzureichend. Kochs Antwort, warum die Aphorismen «zu klassischer Bedeutung gelangt sind», beleuchtet erneut das Verhältnis von Konstantem und Wechselndem in der Geschichte der Medizin:

«So verschieden nun alle diese Antworten aus ihrer Zeitbedingtheit heraus ausfallen müssen, zugleich muß ein viel innerlicherer Grund der dauernden Hochschätzung der Aphorismen bestanden haben und auch heute noch gelten, wenn man sich die Sätze selbst ansieht und wenn man sich vergegenwärtigt, was an der ärztlichen Situation im Wechsel der Zeiten bei allen Fortschritten und Rückschritten im Wissen und Können immer gleich geblieben ist.» ${ }^{41}$

Die Aphorismen hätten wie kaum andere Schriften zum Ausdruck gebracht, was «immer gleich geblieben ist». Sie hätten in unübertroffener Klarheit genau diese Eigenschaften erkannt und beschrieben. Sie lieferten auf die Herausforderungen in der Situation des Helfens das Allgemeingültigste, was es über die Medizin zu sagen gäbe, hier hätten «die schwierigsten, grundlegenden Fragen der Medizin Antworten gefunden [...], die in den reichlich 2000 Jahren seit ihrer Niederschrift im Wechsel der Zeiten und mit dem Wachstum des Wissens selbst lebendig geblieben sind.» ${ }^{42}$ Diese Aussagen seien in-

40 Koch, R. (1933) Warum kamen die hippokratischen Aphorismen zu klassischer Bedeutung? Münchener Medizinische Wochenschrift 80:189-191, S. 190; die einzelnen Interpretationen sind zu finden bei: ders. (1928) Auslegung des zweiten Hippokratischen Aphorismus. Beitrag zur Technik des Unterrichtes in der Geschichte der Medizin. In: Internationale Beiträge zur Geschichte der Medizin. Festschrift zur Feier seines 60. Geburtstages am 8. Dez. 1928 Max Neuburger gewidmet. Wien, S. 209-218; ders. (1930) Auslegung des ersten Hippokratischen Aphorismus. In: Historische Studien und Skizzen zu Natur- und Heilwissenschaft, Festgabe Georg Sticker. Berlin, S. 1-10; ders. (1933) Auslegung des dritten hippokratischen Aphorismus. Archiv für Geschichte der Medizin 26: 281-288; siehe auch Preiser, G. (1988) Richard Kochs Interpretation der hippokratischen Aphorismen. In: Preiser (Hg.) (Anm. 1.), S. 154-161.

41 Koch 1933 (Anm. 40), S. 191.

42 Koch 1933 (Anm. 40), S. 191. 
teressanterweise negativer Art, vor allem im ersten Aphorismus, der treffend wie kaum ein anderes Dokument die grundsätzlichen Schwierigkeiten ärztlichen Tuns benannt hätte.

Wenn Koch von den «Aphorismen mit ihren überzeitlichen Qualitäten» ${ }^{43}$ spricht, dann lässt sich daraus auf die Verlaufsfigur der Geschichte schliessen, denn ein angemessenes Geschichtsbild der Medizin kann nur unter Berücksichtigung ihrer wesenhaften Eigenschaften gefunden werden. Aus der Besinnung auf die Geschichte und aus der Einsicht in die unveränderten Schwierigkeiten ärztlichen Handelns gelte es, «zu der Ewigkeit des Handelns der Urväter zurück[zu]finden» - ja, man habe «die neue Bindung zum Urwesen der Medizin wieder gefunden $»^{44}$. Insofern sieht Koch einen Kern der Medizin, der unverändert ist und bleiben wird; und der Verlauf historischer Ereignisse kann unter dem Aspekt systematisiert werden, in welchem Verhältnis der Bereich des sich Wandelnden zum Kern der Medizin steht. Diese Verlaufsform geschichtlicher Entwicklung entspricht der Einschätzung von Koch, «daß die Gesetze der ärztlichen Strategie ewig, die ihrer Taktik aber abhängig von dem Wissensbestande einer jeden Zeit seien» ${ }^{45}$. Oder, aus anderer Perspektive: Koch ist der festen Überzeugung, dass das diagnostische und therapeutische ärztliche Denken «eine ewige, unbewegliche und eine zeitliche bewegliche Komponente hat. Die unbewegliche tritt uns nun nirgends eindringlicher entgegen, als in dem schriftlich niedergelegten Denken großer Ärzte. $)^{46}$ Mit der letzten Bemerkung hat Koch eine Verbindung zwischen der historischen Verlaufsform der Medizin und einer möglichen Funktion der Medizingeschichte hergestellt. Diese Brücke sei genauer verfolgt.

\section{Funktion der Geschichtsschreibung für eine praktische Wissenschaft}

Das Verhältnis von Konstantem und Veränderlichem in der Geschichte der Medizin lässt sich weiter vertiefen, wenn man den Wirkort betrachtet, den Koch der Medizingeschichtsschreibung zuweist. An die Medizin, genauer: an die praktische Medizin soll sie gebunden werden, und hier soll sie ihre Funktionen entfalten. Koch stellt sich in seiner Antrittsvorlesung demgemäss die Frage, «ob medizingeschichtliche Kenntnisse und medizingeschichtliches Verständnis geeignet sind, dem Arzt in der Ausübung seines Berufes zu nützen» ${ }^{47}$.

43 Koch 1933 (Anm. 40), S. 190.

44 Koch 1927 (Anm. 19), S. 1.

45 Koch 1920/21 (Anm. 38), S. 498.

46 Koch 1920 (Anm. 15), S. 177.

47 Koch 1921 (Anm. 22), S. 217. 
Einer Antwort auf diese Frage sollte Kochs Kritik an der bisherigen Medizinhistoriographie vorausgehen. Zum einen nimmt er das Selbstverständnis der Medizin, zum anderen das Selbstverständnis der Geschichtsschreibung als Ausgangspunkt seiner Kritik: Je mehr sich die Medizin als Naturwissenschaft verstand, umso geringer war ihr Interesse an der Geschichte. Dies lag an der Qualität des Wissens, das an sich und ohne Bezug zur Geschichte wahr sein sollte. Zudem wollte man sich von der «Büchermasse» der Vergangenheit befreien. Dem Arzt - so die Konsequenz - diente die Beschäftigung mit der Vergangenheit einzig «als eine reizvolle antiquarische Beschäftigung für Mußestunden $»^{48}$.

Die Geschichtsschreibung der Medizin reagierte ihrerseits auf diese Wandlung, indem ihr nur noch «die gelehrte Liebe zu der abgeschafften Vergangenheit $»^{49}$ als Motivation diente. Doch ihre Produkte, so sehr Koch deren Gelehrsamkeit rühmt, blieben in der praktischen Medizin wirkungslos. Die Standardwerke der Medizingeschichte - «in die Bildung des Geisteslebens der Aerzte greifen sie bisher kaum ein» ${ }^{50}$. Kurzum: «[...] wegen der Art des medizingeschichtlichen Forschens und wegen des Zustandes der Medizin war es gar nicht möglich, daß Medizingeschichte werden konnte, die selbst zur Medizin gehörte.» ${ }^{51}$

Doch wie kann ein Einfluss auf die praktische Medizin gelingen? Eine Antwort ergibt sich abermals aus der Kritik: Die bisherige Medizinhistoriographie habe - so Koch - das Wesen der Medizin verkannt. Hätte man es zutreffend eingesehen, dann wäre man den Eigenschaften ärztlichen Handelns näher gekommen:

"So sind auch unsere Geschichten der Medizin aus einer gedanklichen Einstellung heraus geschrieben, in der die Empfindung, wie herrlich weit wir es gebracht haben, alle anderen überwiegt. Das, was ihr fehlt, ist die geistige Einstellung des Arztes, dem seine Möglichkeiten, dem Kranken zu helfen, lebenslänglich problematisch bleiben, der sich nicht nur der Stärken, sondern auch der behebbaren und nichtbehebbaren Schwächen seines Könnens dauernd bewußt ist.» ${ }^{52}$

Die Strukturen der Medizin seien massgeblich von den «behebbaren und nichtbehebbaren Schwächen» ärztlichen Handelns im Einzelfall geprägt erinnert sei an die Hippokratischen Aphorismen. Und jeder Arzt, der sich

48 Koch 1928 (Anm. 13), S. 7.

49 Koch 1921 (Anm. 22), S. 218.

50 Koch 1921 (Anm. 22), S. 218. Koch selbst hat nie in diesem Sinne gearbeitet, sondern sich stets der Interpretation historischer Quellen gewidmet.

51 Koch 1921 (Anm. 22), S. 220. In seiner Übersicht zur Funktion der Geschichte in der ärztlichen Ausbildung erwähnt George Rosen die Kritik von Koch, nicht jedoch dessen Antwort auf die missliche Situation; siehe Rosen, G. (1948) The Place of History in Medical Education. Bulletin of the History of Medicine 22: 594-629, insb. 618-619.

52 Koch 1921 (Anm. 22), S. 220. 
dessen bewusst ist und dem seine eigene Tätigkeit «problematisch» bleibe, suche in der Geschichte nach Antworten auf diese Herausforderung:

\begin{abstract}
«Jeder Mensch aber, der eine Tätigkeit ausübt, ist einsam mit sich allein und sucht diese Einsamkeit zu durchbrechen. Dies gilt nicht nur vom Arzt, auch vom Künstler, vom Strategen, vom Politiker. [...] Das ist der erste wirkliche Beziehungspunkt zwischen Medizin und Geschichte, der Drang, die Einsamkeit des Tätigen zu durchbrechen, sich mit anderen, die vor derselben Aufgabe standen in Beziehung zu setzen und zwar am liebsten mit den Größten und Tiefsten. Hier ist lebendiger Drang nach Geschichte, nach Kenntnis klassischer Schriftsteller. Der eine echte Beziehungspunkt zwischen Geschichte und Medizin liegt in der Eigentümlichkeit der Medizin, eine Tätigkeit zu sein.» ${ }^{53}$
\end{abstract}

Nicht nur für den im Einzelfall Handelnden, wie einen Arzt, sondern im Prinzip auch für den Naturforscher treffen diese Aussagen zu. Auch er hat, sofern er sich über seine Situation Klarheit verschafft, einen Drang nach anderen Denkenden: «Wir haben hier einen zweiten Punkt, in dem es eine echte Beziehung zwischen Medizin und Medizingeschichte gibt, im Drang des Denkers nach der Gesellschaft von Denkenden. Und wo soll er diese besser finden als in der Geschichte.» ${ }^{54}$

Einer Medizinhistoriographie, die auf die «Einsamkeit» des im Einzelfall Handelnden Antworten geben kann, schreibt Koch eine weitreichende Funktion zu. Damit befindet er sich im Einklang mit Zeitgenossen, die angesichts der «Krise» von der Geschichtsschreibung kompensatorische Orientierung erwarten. Doch Koch ist geradezu masslos: Es ist «die Geschichte der Medizin, die, richtig ausgenutzt, diese geistige Epidemie zu heilen vermag, die der verblassenden Gestalt des Arztes wieder scharfen Umriß und volle Farben zu geben vermag» ${ }^{55}$, ja die Geschichte der Medizin sei «berufen, die sogenannte Krise in der Medizin zu überbrücken, sie ist die stärkste Waffe im Kampfe gegen unechte Heilkunde» ${ }^{56}$.

\title{
Tradition und Modernität
}

Kochs Bestreben um ein neues Verhältnis zur Vergangenheit ist keineswegs aus verklärender, antimoderner Grundhaltung entstanden, sondern aus Einsichten in den gegenwärtigen Zustand der Medizin. Seine Gedanken zur Kunstgeschichte ${ }^{57}$ bestätigen, dass gerade die Avantgarde als «fortschrittliche» Bewegung mit ihrer Zerstörung des Fortschrittsmythos den Zeitgeist

53 Koch 1921 (Anm. 22), S. 223.

54 Koch 1921 (Anm. 22), S. 223; siehe auch Winau, Rolf (1988) Die Funktion der Medizingeschichte in der Sicht Richard Kochs. In: Preiser (Hg.) (Anm. 1), S. 142-149.

55 Koch 1927 (Anm. 19), S. 2.

56 Koch 1927 (Anm. 19), S. 2.

57 Siehe Anm. 21 
trifft und in diesem Sinne «modern» ist. Ähnliches gilt auch für die Medizin: Nicht der nostalgische Blick in die Vergangenheit, sondern «die Macht der Tatsachen [zwingt] uns [...], daß Alte wieder in unser Leben aufzunehmen ${ }^{58}$.

Aus diesem Grunde hat sich Koch niemals als «Antimoderner» verstanden, im Gegenteil, für ihn verweist die Moderne auf die Vergangenheit, und zwar nicht nur aufgrund geschichtsphilosophischer Überlegungen. Da ein linearer Fortschritt in der Medizin nicht zu erwarten ist, sind es praktische ärztliche Erwägungen, die ein Besinnen auf Vergangenes unterstützen. So belehrt er Aschner, der immer wieder alte Heilverfahren in der Medizin zur Nutzung bringen will: «Er [Aschner] weiß wahrscheinlich selbst nicht, daß er auch im üblichen Sinne moderner ist als der Durchschnitt seiner Kollegen.» ${ }^{59}$ Allein die pragmatische Ausrichtung der Medizin verpflichte dazu, Nützliches für die ärztliche Tätigkeit auch aus deren Geschichte zu entnehmen. «Modernität» ist für Koch in diesem Fall das uneingeschränkte, eklektische Nutzen wirkungsvoller Therapien, egal aus welcher Schule der Medizin sie stammen. Die nicht-lineare Verlaufsform der Geschichte, der Pragmatismus der Medizin und die mögliche Nützlichkeit alter Verfahren zwingen zu einer Rückschau in die Vergangenheit. Dafür sprächen gleichermassen die praktischen Verluste, die durch die «Abstoßung der Vergangenheit» bedingt seien:

«So ging neben vielem anderen die ganze Kunst der Analyse der kranken Persönlichkeit, die individualisierende Diagnostik verloren, ebenso die Theorie der Pathogenese, die Lehre von der Norm, von der Bedeutung der krankhaften Erscheinungen, dann die Lehre von der somatischen Bedingtheit psychischer Krankheitserscheinungen, die physikalische Therapie und die Diätlehre. Das all dies verloren ging, erkennen wir daraus, daß es mittlerweile wieder gefunden wurde. $\rangle^{60}$

\section{Das Judentum und die «Erfindung einer Tradition»}

Kochs Theorie zur geschichtlichen Entwicklung der Medizin - ja der westlichen Kultur schlechthin - ist sichtbar geprägt von seiner Biographie und von den Problemen seines jüdischen Glaubens angesichts der Moderne.Zunächst

58 Koch 1927 (Anm. 19), S. 1.

59 Richard Koch (1931) über den Kreis in der Medizin. Frankfurter Zeitung und Handelsblatt Nr. 441, S. 1. Allerdings kritisiert er Aschners Versuche, alte Theorien, so die Humoralpathologie, «wieder heraufzubeschwören» und als «neue» Theorien gegen die Solidarpathologie anzupreisen. «Dieses Schema ist allzu ärmlich.» (S. 1).

60 Koch 1926 (Anm. 12), S. 290. Eine historische Übersicht zum Argument, die Geschichte sei nützlich für die Medizin, liefert Temkin, O. (1946) An Essay on the Usefullness of Medical History for Medicine. Bulletin of the History of Medicine 19: 9-47. 
zu seiner Biographie: Die Unzulänglichkeiten eines positivistischen Geschichtsverständnisses einzusehen bedeutete auch für Koch eine Abkehr von zuvor fest Geglaubtem und bildete einen Wendepunkt in seiner intellektuellen Entwicklung. Er hat sich dieses Umdenken erst erarbeiten müssen und bekennt in seinen Lebenserinnerungen, dass in seinem Bezugsfeld $\mathrm{zu}$ Jugendzeiten «alle fest davon überzeugt waren, daß keine Vergangenheit vor der augenblicklichen neuen Zeit bestehen konnte. Das war uns so selbstverständlich, daß wir uns darüber garnicht den Kopf zerbrachen.» ${ }^{61}$

Überdies ist sein Geschichtsverständnis nicht ohne den Bezug zum jüdischen Glauben zu verstehen. Auch wenn Koch an keiner Stelle seiner publizierten Werke ${ }^{62}$ eine Verbindung zwischen seinen Ideen vom zeitgemässen Judentum und seiner Medizintheorie herstellt, so lassen sich gleichwohl Gemeinsamkeiten benennen: Kochs Suche nach einem akzeptablen Geschichtsbild in der Medizin gleicht der Suche vieler jüdischer Zeitgenossen des frühen 20. Jahrhunderts nach einem authentischem Judentum und einem ebensolchen Verhältnis zur jüdischen Überlieferung. Die Aussenbedingungen sind ähnlich: Wie ist eine Geschichte, eine Tradition, ein historisches Selbstverständnis in einer sich rapide wandelnden Welt zu finden, wenn eine völlige Abkehr von der Vergangenheit ebensowenig möglich ist wie eine unkritische, gar vollständige Übernahme von Vergangenem? Wie gestaltet sich unter modernen Bedingungen eine authentische jüdische Tradition - zumal unter dem Druck einer überwiegend christlichen Gesellschaft? Genau mit dieser Frage sahen sich gläubige jüdische Intellektuelle konfrontiert, nicht nur in der Weimarer Republik, sondern bereits in der reformerischen Tradition und der Wissenschaft vom Judentum ${ }^{63}$.

Richard Koch stand als Dozent am Frankfurter «Freien Jüdischen Lehrhaus», der bedeutendsten Institution der jüdischen Erwachsenenbildung in der Weimarer Republik, in engem Kontakt mit Franz Rosenzweig (1886-1929) und Martin Buber (1878-1965) und war befreundet mit Eduard Strauss (1876-1952). Koch hat an der intellektuellen Suche mitgewirkt und

61 Koch, R. (1943-48) Ohne Titel [Lebenserinnerungen]. Manuskript, Maschinenschrift, unveröffentlicht, 3 Bände. 1. Band, S. 187. Den Hinweis auf die Lebenserinnerungen verdanke ich Prof. Dr. Dr. Rolf Winau, Berlin; das Manuskript hat mir freundlicherweise Prof. Dr. Walter Laqueur, Washington, überlassen.

62 Eine Analyse der zahlreichen unpublizierten Schriften Kochs unter dieser Fragestellung muss einer gesonderten Untersuchung vorbehalten bleiben, da die weit verstreuten Bestände noch nicht einmal vollständig gesichtet sind.

63 Brenner, M. (1996) The Renaissance of Jewish Culture in Weimar Germany. New Haven, London. 
an einer zentralen Stelle der «Jüdischen Renaissance», wie Buber es nannte, mitgearbeitet ${ }^{64}$; er kannte die Frage seiner Glaubensgemeinschaft nach einem authentischen Verhältnis zur Vergangenheit.

Auch die jüdischen Intellektuellen gaben eine Antwort, bei der Wechselndes ebenso wie Kontinuierliches unter neuen Bedingungen und in Abwandlungen in ein rechtes Verhältnis zu rücken waren. Brenner fasst das Ergebnis wie folgt zusammen: «Jewish culture in Weimar Germany was characterized neither by a radical break with the past nor by a return to it. Indeed, it used distinct forms of Jewish traditions, marking them as authentic, and presented them according to the demands of contemporary taste and modern cultural forms of expression. ${ }^{65}$ Brenner betont die notwendig konstruktiven Züge dieser Suche nach einem neuen Verhältnis zur Vergangenheit: «What seems to be a retrieval of authentic traditions becomes, rather, a modern construction or invention of tradition.» ${ }^{66}$

\section{Diskussion}

$\mathrm{Ob}$ es Richard Koch gelungen ist, eine solche Tradition für die Medizin zu «erfinden», mag dahingestellt sein, allein weil sein Werk nicht vollendet wurde. An den Ausführungen von Koch bliebe überdies in systematischer Hinsicht vieles zu kritisieren: Seine fast ubiquitäre Verwendung von «lebendig» ist wenig erklärend, wenn er beispielsweise von der «lebendigen» Geschichte, der «lebendigen» Arzt-Patient-Beziehung, von einer «lebendigen» Geschichtsschreibung, von einem «lebendigen» Verhältnis zur Geschichte und einem «lebendigen» Drang nach Geschichte spricht. Diese Eigenschaften liessen sich vermutlich differenzierter beschreiben; jedenfalls stiftet ihre Bezeichnung mit einem einzigen Adjektiv eher Unklarheit. Ferner könnte man Koch vorwerfen, dass er einem «genetischen Fehlschluß» erlegen sei. Seine Hoffnung, in den Anfängen finde sich das Wesenhafte der Medizin, das auch heute noch Relevanz besitze, müsste durch theoretische Argumente gestützt werden. Diese zu liefern unterlässt Koch jedoch.

64 Koch, R. (1923) Das Freie Jüdische Lehrhaus in Frankfurt am Main. Der Jude 7: 116-127.

65 Brenner 1996 (Anm. 64), S. 5.

66 Brenner 1996 (Anm. 64), S. 4;Volkov,S. (1991) Die Erfindung einer Tradition. Zur Entstehung des modernen Judentums in Deutschland. Historische Zeitschrift 253: 603-628, schreibt diesen Bemühungen eine herausragende Stellung in der jüdischen Kultur zu: «Die Erfindung der Tradition war das einzige, jedoch äußerst wichtige jüdische «Projekt der Moderne〉.» (S. 628). 
Sein historischer Rückgriff auf die biologischen Eigenschaften der Organismen verbleibt überdies bei einer ganz bestimmten historischen Vorstufe, und Koch begründet nicht, warum er nicht weiter zurückgeht und die biologischen Eigenschaften selbst historisiert. Die anthropologischen und biologischen Konstanten, auf die er sich bei der Beschreibung des Unveränderlichen bezieht, werden seinerseits nicht weiter hinterfragt; sie werden nicht historisiert, also auch nicht als kontingent beschrieben. Insofern gelangt auch Koch bei seiner Suche in der Geschichte zu einem Ergebnis jenseits der Geschichte.

Gleichermassen hinterfragenswert ist die beträchtliche Funktion, die er der Geschichte zuschreibt, wenn er fordert, «daß die Zukunft an der Vergangenheit genesen muß, wenn wir nicht das Erkalten unserer Sonne im Geiste vorwegnehmen wollen.» ${ }^{67}$ Nietzsches Befürchtungen zum Historismus versucht er zu entkräften, in dem er entgegensetzt: «Was wir heute Historismus in der Medizin nennen dürften, wäre keine Anleihe bei der Vergangenheit, sondern eine Zwiesprache mit ihr.» ${ }^{68}$ Schaut man sich das vorherige Zitat an, so bleibt zweifelhaft, ob er Nietzsches Kritik damit entkräftet hat. Kochs hohe Funktionszuweisung an die Geschichte, das «Genesen der Zukunft an der Vergangenheit» ist sicher nicht in Nietzsches Sinne ${ }^{69}$.

Doch man wird den Intentionen des Autors nicht gerecht, kritisiert man seine Unzulänglichkeiten in systematischer oder geschichtsphilosophischer Hinsicht. Denn offensichtlich war Koch gar nicht daran gelegen, eine abgeschlossene Geschichtsphilosophie zu schreiben. An keiner Stelle seines Werkes hat er sich dieser Aufgabe explizit gestellt; es sind allenfalls zahlreiche Bemerkungen in seinen Publikationen zu finden. Bis auf die Frage, welche Funktion der Geschichtsunterricht für Medizinstudenten einnimmt, ist ihm der geschichtstheoretische Gegenstand eher ein Folgeproblem, wenn auch ein bedeutendes. Kochs Sichtweise ist vornehmlich von seiner epistemologischen Frage an die Medizin geprägt. Hier verfolgt er tatsächlich systematischen Anspruch und entwickelt eine eigenständige Position.

Richard Koch hat sich stets als Aussenseiter unter seinen medizinhistorischen Kollegen empfunden. Trotz seiner Verehrung und freundschaftlichen Beziehung zu Karl Sudhoff (1853-1938), trotz seiner Studien bei Julius Leopold Pagel (1851-1912) war er sich doch immer seines eigenständigen An-

67 Koch 1927 (Anm. 19), S. 1.

68 Koch 1926 (Anm. 12), S. 297.

69 Vgl. Nietzsche, F. (1994) Unzeitgemäße Betrachtungen, Zweites Stück: Vom Nutzen und Nachteil der Historie für das Leben. In:Werke in drei Bänden, hrsg. von K. Schlechta. Darmstadt, Bd. 1, S. 209-286. 
satzes bewusst ${ }^{70}$. Er hat sich gleichermassen einer Bindung an eine Schule der allgemeinen Geschichtswissenschaften oder an eine zeitgenössische philosophische Theorie verweigert, abgesehen von der deutlichen Bezugnahme auf Hans Vaihingers (1852-1933) «Philosophie des Als Ob» ${ }^{71}$. Auf das umfangreiche Schriftgut der Deutschen Historischen Schule geht er nur insofern ein, als dass er in seiner Antrittsvorlesung eine terminologische Unterscheidung von Windelband und Rickert übernimmt ${ }^{72}$.

Kochs mangelnde Bezugnahme auf andere Disziplinen erklärt sich insofern, als dass ihm immer daran gelegen war, von der praktischen Medizin und nicht von einer anderen Disziplin her zu argumentieren ${ }^{73}$. Sein Geschichtsbild ist, wie auch seine übrige Medizintheorie, ganz von der Perspektive dieser Disziplin und von ihrer Eigenart als praktische Tätigkeit bestimmt. Demgemäss hat Koch die Geschichtsschreibung der Medizin immer ganz eng an die praktische ärztliche Tätigkeit binden wollen. Er verstand sein Fach nicht als ein Spezialgebiet der allgemeinen Geschichtswissenschaften, sondern als ein Spezialgebiet der praktischen Medizin; seine argumentative Stärke und Originalität liegt vor allem in der Funktionsbestimmung der Geschichte angesichts der praktischen Orientierung der Medizin ${ }^{74}$. Diese Tendenz schlägt sich auch in der Themenwahl nieder: Koch widmet sich der Sozialgeschichte nur ganz nebensächlich; er verbleibt bei Themen, die die ärztliche Tätigkeit

70 «[... ich hatte während meiner ganzen Lehrjahre nicht das Glück mit einem einzigen Lehrer in enge Beziehung zu treten, der mir Sinn, Bedeutung, Schönheit, Ethos, Pathos, Eros der feststellenden und beschreibenden oder auch nur der reinen Wissenschaft und ihrer Methoden erschlossen hätte. [...] Aber mir scheint, daß ich im Letzten und Höchsten Grund habe, diesem Schicksal zu danken, welches mich mit fester Hand einen anderen Weg des Geistes führte.» (Koch 1943-48 [Anm. 62], Band 3, S. 26). Den Unterschied zu Pagels Vorstellungen beschreibt Koch 1928 (Anm. 13), S. 11; siehe auch Pagel, W. (1951) Julius Pagel and the Significance of Medical History for Medicine. Bulletin of the History of Medicine 25: 207-225.

71 Koch, R. (1924) Das Als-Ob im ärztlichen Denken. München; vgl. Vaihinger, H. (1927) Die Philosophie des Als Ob. Leipzig, 9./10. Aufl.

72 Auch andere führende Medizinhistoriker haben - wenn es um die Legitimation ihres Faches ging - auf die allgemeinen Geschichtswissenschaften keinen Bezug genommen. «Sie waren ganz darauf konzentriert, die Medizingeschichte gegenüber der naturwissenschaftlichen Medizin zu rechtfertigen.» (Kümmel, W. F. (1997) Vom Nutzen eines «nicht notwendigen Faches»: Karl Sudhoff, Paul Diepgen und Henry E. Sigerist vor der Frage «Wozu Medizinschichte?». Erscheint in R. Toellner und U. Wiesing (Hg.) Geschichte und Ethik in der Medizin. Stuttgart, im Druck).

73 «Die Philosophie der Medizin kann heute nicht auf die akademische Philosophie, auch nicht auf die Philosophie der Naturwissenschaften aufgebaut werden, sondern nur auf die Tatsachen der Medizin im engsten Sinne des Wortes.» (Koch 1928 [Anm. 13], S. 15).

74 Hier unterscheidet er sich auch von Karl Sudhoff, Paul Diepgen (1878-1966) und Henry E. Sigerist (1891-1957); vgl. Kümmel 1997 (Anm. 72). 
aus ihrer Innenperspektive heraus beleuchten ${ }^{75}$. Die Geschichte des ärztlichen Denkens und deren bedeutende Personen interessieren ihn.

Koch war klug genug, die Vereinfachungen seiner Zeitgenossen zur Geschichte der Medizin nicht zu wiederholen ${ }^{76}$. Vor allem verzichtet er auf verklärende Verkündungen des «ewigen Arztes», wiewohl er immer wieder erklärt, dass sich in wesentlichen Fragen der Medizin nur Unwesentliches verändert habe. Grund dafür sind die Herausforderungen und Unwägbarkeiten, denen sich eine Disziplin gegenüber sieht, die es sich zur Aufgabe gemacht hat, kranken Menschen zu helfen. Als Argument für die Konstanz der Herausforderungen führt Koch die zeitlich weithin unveränderte biologische Beschaffenheit des Menschen an; die Medizin findet in den spontanen krankheitsabwehrenden Reaktionen der Organismen ihren Vorläufer. Die hohe Konstanz im Verlauf der Geschichte erklärt auch, warum Koch das Hippokratische Motto seiner wichtigsten Monographie voranstellt: «Die Heilkunde hat von jeher alles» ${ }^{77}$.

Bei Koch ist der geschichtliche Verlauf der Medizin nicht durch das stetig ansteigende Wissen geprägt, sondern durch Wechselndes und Unverändertes angesichts der ständigen Herausforderungen ärztlichen Handelns. Der epistemologische Status als Tätigkeit, die aufhebbaren und nicht aufhebbaren Schwierigkeiten ärztlichen Tuns und die Einsamkeit des Handelnden kurz: die strukturellen Bedingungen ärztlichen Handelns sind weitgehend konstant geblieben. Hier sieht Koch die Brücke zur Geschichte der Medizin. Wer sich dieser Bedingungen bewusst ist, wer die «Einsamkeit» angesichts der Herausforderungen und Unwägbarkeiten seines Tuns erkennt, den «drängt» es nach Geschichte, weil er hier Antworten finden kann. Unter dieser Einsicht kann die Medizingeschichte für den praktischen Arzt eine fruchtbare Funktion übernehmen: «Wenn die Medizin sich ihrer eigenen Gesetze wieder bewußt geworden ist, dann wird auch ein Gebiet der Heilkunde, das heute ein sonderbar lebensfremdes Dasein führt, die Geschichte der Heilkunde, zu neuem Leben erwachen.» ${ }^{78}$

75 Gegen Ende seines Lebens, im sowjetischen Exil und unter dem Eindruck der politischen und menschlichen Katastrophe, schreibt Koch seinem Kollegen Sigerist, dass er die politische Dimension ärztlichen Handelns wohl unterbewertet habe: «Als wir jung waren, konnten wir noch nicht sehen, in welchem Maß das Handeln des Arztes, der Krankheitsbegriff und alle anderen gedanklichen und manuellen Gebiete der Medizin eine Funktion der Gesellschaftsordnung und der Weltanschauung sind, eine Erkenntnis, die Ihnen [H.E. Sigerist] früher in den Mittelpunkt der Betrachtung rückte als mir.» (Brief an Henry E. Sigerist, undatiert (März/April 1949), Yale University Library, New Haven, Connecticut).

76 Siehe Wiesing 1996 (Anm. 11).

77 So auf dem Titelblatt der «ärztlichen Diagnose» (Anm. 15) in beiden Auflagen.

78 Koch 1920 (Anm. 15), S. 176. 
Kochs Äusserungen beinhalten eine herbe Kritik am geschichtlichen Selbstverständnis der Medizin im 19. Jahrhundert. Diese Epoche habe keinesfalls das eigentliche Selbstverständnis der Medizin beschrieben, sondern das «Urwesen» der Medizin verkannt. Sie sei nicht der Ort, an dem man die Medizin endgültig und wahrhaft definiert habe, sondern in epistemologischer wie geschichtstheoretischer Hinsicht eine Verfehlung.

Unbesehen aller systematischen Einwände bleibt Kochs Theorie bedenkenswert, weil sie eine Verbindung zwischen den Eigenschaften der praktisch-ärztlichen Tätigkeit und der Geschichte herstellt. Koch hat auch in dieser Hinsicht keine abgeschlossene Theorie eines historischen Selbstverständnisses der Medizin zurückgelassen. Er hat jedoch ohne Zweifel die Problematik erkannt und wichtige Elemente einer Antwort auf die Frage geliefert, was es für die Medizin als praktische Wissenschaft heisst, in Zeiten des hastigen Wandels eine Geschichte zu haben. 